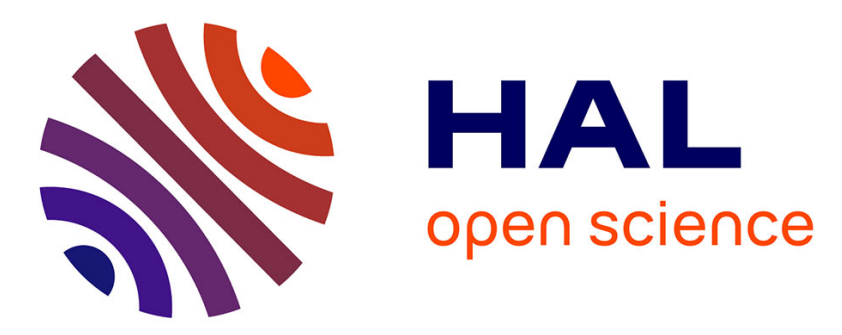

\title{
Bounded Attitude Stabilization: Real-Time Application on Four-Rotor Mini-Helicopter
}

Fermi Guerrero Castellanos, Nicolas Marchand, Suzanne Lesecq, Jérôme Delamare

\section{- To cite this version:}

Fermi Guerrero Castellanos, Nicolas Marchand, Suzanne Lesecq, Jérôme Delamare. Bounded Attitude Stabilization: Real-Time Application on Four-Rotor Mini-Helicopter. IFAC WC 2008 - 17th IFAC World Congress, Jul 2008, Séoul, South Korea. pp.3167-3173, 10.3182/20080706-5-KR-1001.00538 . hal-00200555

\section{HAL Id: hal-00200555 https://hal.science/hal-00200555}

Submitted on 18 Apr 2013

HAL is a multi-disciplinary open access archive for the deposit and dissemination of scientific research documents, whether they are published or not. The documents may come from teaching and research institutions in France or abroad, or from public or private research centers.
L'archive ouverte pluridisciplinaire HAL, est destinée au dépôt et à la diffusion de documents scientifiques de niveau recherche, publiés ou non, émanant des établissements d'enseignement et de recherche français ou étrangers, des laboratoires publics ou privés. 


\title{
Bounded attitude stabilization: Real-time application on four-rotor mini-helicopter *
}

\author{
J. F. Guerrero-Castellanos * N. Marchand* S. Lesecq* \\ J. Delamare ${ }^{* *}$ \\ * GIPSA-lab, Control Systems Department (former LAG). \\ ** G2Elab, Electrical Engineering Department \\ CNRS-INPG-UJF, ENSIEG BP 46, 38402 St Martin d'Hères-Cedex, \\ France, \\ (Tel: +33-(0)4-76-82-64-13; e-mail: fergue@lag.ensieg.inpg.fr)
}

\begin{abstract}
This paper deals with the global stabilization of a rigid body by means of a bounded quaternion-based feedback. In addition to input bounds, the nonlinear control takes into account the slew rate limits. The proposed control scheme is generic and can be applied to all systems falling in the framework of rigid bodies. Furthermore, its extreme simplicity rendering it suitable for embedded implementation. It is also shown that the control strategy can be applied to attitude stabilization of a four-rotor mini-helicopter despite gyroscopic effects appear that are not present in the rigid body framework. Several real-time experiments have been performed on a real mini-helicopter in order to show the effectiveness of the proposed control approach.
\end{abstract}

\section{INTRODUCTION}

The problem of attitude stabilization of a rigid body has been a subject that has attracted considerable amount of interest over the past 30 years. Several approaches have been applied such as feedback linearizing control, Wie et al. (1989), Fjellstad and Fossen (1994), feedback proportional-derivative control, Wen and Kreutz-Delgado (1991), Joshi et al. (1995), predictive control in Wen et al. (1997) and Hegrenas et al. (2005)), integral backstepping in Kristiansen and Nicklasson (2005) and robust control applied to tactical missiles, Song et al. (2005). This list is not exhaustive. Within these mentioned approaches, the feedback linearizing control and the feedback proportionalderivative control are widely applied. This is due to the fact that these control laws achieve global stabilization and they are simples to implement. The major criticism of the two last approaches is that for large attitude or angular velocity error, the control law may yield a control input that the actuators are unable to produce. This fact becomes apparent due to their linear nature. In practice, limitations on available energy impose bounded input signal. Moreover, it is common that due to sensors limitation the output of the system are bounded. Actually, the above cited attitude control approaches do not consider the problem which takes the input constraints into account. Few publications have dealt with this problem.

In Tsiotras and Luo (2000), a saturating control law for the kinematic system of an under-actuated rigid spacecraft is studied. This control law uses a non-standard attitude representation, which allows the decomposition of general motion into two rotations. In Belta (2004), a control law that drives a rigid underwater vehicle between arbitrary initial and final region of the state space while satisfying bounds on control and state is proposed. The approach is based in a control of multi-affine systems.

\footnotetext{
‡ This work was supported in part by CONACYT-Mexico
}

The authors in Boskovic et al. (1999) have studied the robust sliding mode stabilization of the spacecraft attitude dynamics in presence of control input saturation based on the variable structure control (VSC) approach. The application of optimal control to attitude problem of a rigid body has been also the interest of many research, (see Scrivener and Thompson (1994) and the references therein). In order to circumvent the solution of HamiltonJacobi equations, different works have used the so-called inverse optimal method, Osipchuck et al. (1997), Krstić and Tsiotras (1999). Unfortunately, the above cited methods are either non smooth or computationally expensive (optimal control). This renders difficult their practical implementation.

In the present work a bounded control law for the global attitude stabilization of a rigid body is proposed. With the proposed approach, the control bounds along the three axis can be completely freely chosen. The bounded control law can force the closed-loop angular velocity to any a priori specified region of the state space and maintains them in it thereafter. Therefore, in addition to input constraints, the control law enforces angular velocity constraints, i.e. slew rate limits. Hence, the control law is robust towards bounded angular velocities measurements as it happens with classical rate gyros. Furthermore, the control law is robust towards modelling errors because it does not depend on the knowledge of the inertial parameters of the system. The proposed control is inspired by the nested saturation functions approach proposed by Teel (1992) for the problem of stabilizing linear systems with bounded controls. The attitude is parameterized using the unit quaternion, which allows nonlinear large angles attitude manoeuvres. This makes a distinct contrast from the case using three parameters representation, where an inherent geometric singularity appear (see Shuster (1993) for more details on attitude representations). The principal property of proposed control law is its extreme simplicity com- 
pared to the other existing approaches, rendering it very suitable for embedded implementation, where on-board computational resources are challenging. Furthermore, the control signal computed with this control law is smooth. In recent years, significant research effort has been directed toward the control, modelling and design of flying robots. This interest arise principally of military purposes but also of civilian application such as disaster monitoring, environmental surveillance or even cinematography. Within flying robots, the four-rotor helicopter has aroused great interest because of its highly manoeuvrability and the ability to hover. This Vertical Take-Off and Landing (VTOL) vehicle has some advantages over conventional helicopters: owing to symmetry, this vehicle is simple to design and construct. The low moment of inertia of this aerial robot makes it vulnerable to large angular acceleration. Therefore, for many potential missions an efficient attitude control is crucial, since it allows the vehicle to maintain a desired orientation. Several control techniques have been used for the attitude stabilization of the four-rotor helicopter, for instance, PID and LQ control in Bouabdallah et al. (2004), sliding mode control in Bouabdallah and Siegwart (2005) and Backstepping approach applied in Tayebi and McGlivary (2006). Although the results presented in the above cited works are nice and founding, the boundedness of the control input is not considered.

One particular aspect in the four-rotor helicopter dynamics, is that the dynamical model for the attitude may be reduced to this one of a rigid body. Therefore, the proposed approach for the stabilization of a rigid body, is naturally extended to the attitude stabilization of the four-rotor mini helicopter. Owing to the extreme simplicity and the smoothness of the control approach, a real-time stabilization was performed successfully on the four-rotor mini helicopter, developed at gipsa-lab control system department (former LAG) in Grenoble. The control capabilities were tested for different scenarios, showing a good performance in terms of settling time stabilization, robustness towards disturbance rejection and tracking trajectories capability. This paper extends the initial work proposed in GuerreroCastellanos et al. (2007) by proposing a control law with more tuning parameters, established robustness properties and a real-time implementation.

The present paper is organized as follows. In section 2, a rigid body quaternion-based orientation is given. Then the main problem is formulated in section 3. The section 4 is devoted to the control law design, where its stability is proved and its properties are presented. The extension of the proposed control law for the attitude stabilization of the four-rotor helicopter is demonstrated in section 5 . Then, the implementation and experimental results are presented and discussed in section 6 .

\section{MATHEMATICAL BACKGROUND}

The attitude of a rigid body can be represented by a quaternion, consisting of a unit vector $\boldsymbol{e}$, known as the Euler axis, and a rotation angle $\beta$ about this axis. The quaternion $q$ is then defined as follows

$$
q=\left(\begin{array}{c}
\cos \frac{\beta}{2} \\
e \sin \frac{\beta}{2}
\end{array}\right)=\left(\begin{array}{c}
q_{0} \\
\boldsymbol{q}
\end{array}\right) \in \mathbb{H}
$$

where

$$
\mathbb{H}=\left\{q \mid q_{0}^{2}+\boldsymbol{q}^{T} \boldsymbol{q}=1, q=\left[\begin{array}{ll}
q_{0} & \boldsymbol{q}
\end{array}\right]^{T}, q_{0} \in \mathbb{R}, \boldsymbol{q} \in \mathbb{R}^{3}\right\}
$$

$\boldsymbol{q}=\left[\begin{array}{lll}q_{1} & q_{2} & q_{3}\end{array}\right]^{T}$ and $q_{0}$ are known as the vector and scalar parts of the quaternion respectively. In attitude control applications, the unit quaternion represents the rotation from an inertial coordinate system $N\left(x_{n}, y_{n}, z_{n}\right)$ located at some point in the space (for instance, the earth NED frame), to the body coordinate system $B\left(x_{b}, y_{b}, z_{b}\right)$ located at the center of mass of the rigid body. The rotation matrix $C(q)$ corresponding to the attitude quaternion $q$, is computed as $C(q)=\left(q_{0}^{2}-\boldsymbol{q}^{T} \boldsymbol{q}\right) I_{3}+2\left(\boldsymbol{q} \boldsymbol{q}^{T}-q_{0}\left[\boldsymbol{q}^{\times}\right]\right)$. where $I_{3}$ is the identity matrix and $\left[\xi^{\times}\right]$is the skew symmetric tensor associated with the axial vector $\xi$.

Denoting by $\boldsymbol{\omega}=\left[\begin{array}{lll}\omega_{1} & \omega_{2} & \omega_{3}\end{array}\right]^{T}$ the angular velocity vector of the body frame $B$ relative to the inertial frame $N$, expressed in $B$, the kinematics equation is given by

$$
\begin{aligned}
\left(\begin{array}{c}
\dot{q}_{0} \\
\dot{\boldsymbol{q}}
\end{array}\right) & =\frac{1}{2}\left(\begin{array}{c}
-\boldsymbol{q}^{T} \\
I_{3} q_{0}+\left[\boldsymbol{q}^{\times}\right]
\end{array}\right) \boldsymbol{\omega} \\
& =\frac{1}{2} \Xi(q) \boldsymbol{\omega}
\end{aligned}
$$

The attitude error is used to quantify the mismatch between two attitudes. If $q$ defines the current attitude quaternion and $q_{d}$ is the reference quaternion, i.e. the desired orientation, then the error quaternion that represents the attitude error between the current orientation and the desired one is given by

$$
q_{e}=q \otimes q_{d}^{-1}
$$

$\otimes$ denotes the quaternion multiplication and $q_{d}^{-1}$ is the complementary rotation of the quaternion $q_{d}$, which is the quaternion conjugate (see (Shuster (1993)) for more details).

The attitude dynamics of a rigid body is described by

$$
J \dot{\omega}=-\omega \times J \boldsymbol{\omega}+\Gamma
$$

where $J \in \mathbb{R}^{3 \times 3}$ is the symmetric positive definite constant inertial matrix of the rigid body expressed in the $B$ frame and $\Gamma \in \mathbb{R}^{3}$ is the vector of control torques. Note that these torques also depend on the environmental disturbance torques (aerodynamic, gravity gradient, etc.).

\section{PROBLEM STATEMENT}

The objective is to design a control law that drives the rigid body attitude to a specified constant orientation and maintains this orientation. Let $q_{d}$ denote the desired constant rigid body orientation, then the control objective is described by the following asymptotic conditions

$$
q \rightarrow q_{d}, \quad \boldsymbol{\omega} \rightarrow 0 \text { as } t \rightarrow \infty
$$

The quaternion error that represents the attitude error between the current orientation and the desired one is given by equation (4). If the the desired attitude is $q_{d}=$ $\left[\begin{array}{llll} \pm 1 & 0 & 0 & 0\end{array}\right]^{T}$ (inertial coordinate frame), the quaternion error (4) coincides with the current attitude quaternion, that is, $q_{e}=q$. This control objective is then

$$
q \rightarrow\left[\begin{array}{llll} 
\pm 1 & 0 & 0 & 0
\end{array}\right]^{T}, \quad \boldsymbol{\omega} \rightarrow 0 \text { as } t \rightarrow \infty
$$

In this study the case $q_{d}=\left[\begin{array}{llll}1 & 0 & 0 & 0\end{array}\right]^{T}$ that represents the attitude aligned up with the inertial coordinate system axes is considered. Nevertheless, the results can be applied to another desired orientation.

It is well known that actuator saturation reduces the 
benefits of the feedback. When the controller continuously outputs infeasible control signals that saturate the actuators, system instability may follow. Therefore, besides the asymptotic stability, the objective of the control law is to take into account the physical constraints of the control system, in order to apply only feasible control signals to the actuators.

\section{BOUNDED ATTITUDE CONTROL FORMULATION}

In this section, a control law that stabilizes the system described by (3) and (5) is proposed. The goal is to design a control torque that is bounded.

Definition 1. Given a positive constant $M$, a continuous, nondecreasing function $\sigma_{M}: \mathbb{R} \rightarrow \mathbb{R}$ is defined by

(1) $\sigma_{M}(s)=s$ if $|s|<M$;

(2) $\sigma_{M}(s)=\operatorname{sign}(s) M$ elsewhere;

Theorem 1. Consider the rigid body rotational dynamics described by (3) and (5) with the following bounded control inputs $\Gamma=\left[\Gamma_{1}, \Gamma_{2}, \Gamma_{3}\right]^{T}$ such that

$$
\Gamma_{i}=-\alpha_{i} \sigma_{M_{i}}\left(\lambda_{i}\left[\omega_{i}+\rho_{i} q_{i}\right]\right)
$$

with $i \in\{1,2,3\}$ and where $\sigma_{M_{i}}$ are saturation functions with $M_{i} \geq 3 \lambda_{i} \rho_{i} . \alpha_{i}, \lambda_{i}$ and $\rho_{i}$ are positive parameters. Then the inputs (8) globally asymptotically stabilize the rigid body to the origin $\left(q_{0}=1, \boldsymbol{q}=0\right.$ and $\left.\boldsymbol{\omega}=0\right)$.

Proof. Consider the candidate Lyapunov function $V$, which is positive definite, radially unbounded and which belongs to the class $C^{2} . V$ represents the total energy of the system

$$
\begin{aligned}
V & =\frac{1}{2} \boldsymbol{\omega}^{T} J \boldsymbol{\omega}+\kappa\left(\left(1-q_{0}\right)^{2}+\boldsymbol{q}^{T} \boldsymbol{q}\right) \\
& =\frac{1}{2} \boldsymbol{\omega}^{T} J \boldsymbol{\omega}+2 \kappa\left(1-q_{0}\right)
\end{aligned}
$$

where $J$ is defined as before, and $\kappa>0$ must be determined. The derivative of (9) after using (3) and (5) is given by

$$
\begin{aligned}
\dot{V}= & \boldsymbol{\omega}^{T} J \dot{\boldsymbol{\omega}}-2 \kappa \dot{q}_{0} \\
= & \boldsymbol{\omega}^{T}(-\boldsymbol{\omega} \times J \boldsymbol{\omega}+\Gamma)+\kappa \boldsymbol{q}^{T} \boldsymbol{\omega} \\
= & \underbrace{\omega_{1} \Gamma_{1}+\kappa q_{1} \omega_{1}}_{\dot{V}_{1}}+\underbrace{\omega_{2} \Gamma_{2}+\kappa q_{2} \omega_{2}}_{\dot{V}_{2}} \\
& +\underbrace{\omega_{3} \Gamma_{3}+\kappa q_{3} \omega_{3}}_{\dot{V}_{3}}
\end{aligned}
$$

$\dot{V}$ is the sum of three terms $\left(\dot{V}_{1}, \dot{V}_{2}, \dot{V}_{3}\right)$. First $\dot{V}_{1}$ is analyzed. From $\Gamma_{1}$ in (8) and equation (10), one gets

$$
\dot{V}_{1}=-\alpha_{1} \omega_{1} \sigma_{M_{1}}\left(\lambda_{1}\left[\omega_{1}+\rho_{1} q_{1}\right]\right)+\kappa q_{1} \omega_{1}
$$

Assume that $\left|\omega_{1}\right|>2 \rho_{1}$, that is $\left.\left|\omega_{1}\right| \in\right] 2 \rho_{1},+\infty[$. Since $\left|q_{1}\right| \leq 1$, it follows that $\left|\omega_{1}+\rho_{1} q_{1}\right| \geq \rho_{1}+\epsilon$ for any $\epsilon>0$ sufficiently small. Therefore, $\omega_{1}+\rho_{1} q_{1}$ has the same sign as $\omega_{1}$. From equation (11) and the norm condition on the quaternion, $\dot{V}_{1}$ takes the following form

$$
\begin{aligned}
\dot{V}_{1} & =-\alpha_{1} \omega_{1} \sigma_{M_{1}}\left(\lambda_{1}\left[\omega_{1}+\rho_{1} q_{1}\right]\right)+\kappa \omega_{1} q_{1} \\
& \leq-\alpha_{1}\left|\omega_{1}\right| \sigma_{M_{1}}\left(\lambda_{1}\left(\rho_{1}+\epsilon\right)\right)+\kappa\left|\omega_{1}\right|
\end{aligned}
$$

Taking

$$
\kappa<\alpha_{1} \min \left(M_{1}, \lambda_{1} \rho_{1}+\epsilon\right)
$$

one can assure the decrease of $V_{1}$ i.e. $\dot{V}_{1}<0$. Consequently, $\omega_{1}$ enters $\Phi_{1}=\left\{\omega_{1}:\left|\omega_{1}\right| \leq 2 \rho_{1}\right\}$ in finite time $t_{1}$ and remains in it thereafter. In this case, $\left(\omega_{1}+\rho_{1} q_{1}\right) \in$ $\left[-3 \rho_{1}, 3 \rho_{1}\right]$.

Let $M_{1}$ verify the following inequality $M_{1} \geq 3 \lambda_{1} \rho_{1}$, equation (13) then becomes:

$$
\kappa<\alpha_{1} \lambda_{1} \rho_{1}+\epsilon
$$

For $t_{2}>t_{1}$, the argument of $\sigma_{M_{1}}$ will be bounded as follows

$$
\left|\lambda_{1}\left(\omega_{1}+\rho_{1} q_{1}\right)\right| \leq 3 \lambda_{1} \rho_{1} \leq M_{1}
$$

Consequently, $\sigma_{M_{1}}$ operates in a linear region

$$
\Gamma_{1}=-\alpha_{1} \lambda_{1}\left[\omega_{1}+\rho_{1} q_{1}\right]
$$

As a result, (11) becomes

$$
\dot{V}_{1}=-\alpha_{1} \lambda_{1} \omega_{1}^{2}-\alpha_{1} \lambda_{1} \rho_{1} \omega_{1} q_{1}+\kappa \omega_{1} q_{1}
$$

Choosing $\kappa=\alpha_{1} \lambda_{1} \rho_{1}$ which satisfies inequality (14), one obtains

$$
\dot{V}_{1}=-\alpha_{1} \lambda_{1} \omega_{1}^{2} \leq 0
$$

The same argument is applied to $\dot{V}_{2}$ and $\dot{V}_{3}$, (10) becomes

$$
\begin{aligned}
\dot{V} & =\dot{V}_{1}+\dot{V}_{2}+\dot{V}_{3} \\
& =-\left(\alpha_{1} \lambda_{1} \omega_{1}^{2}+\alpha_{2} \lambda_{2} \omega_{2}^{2}+\alpha_{3} \lambda_{3} \omega_{3}^{2}\right) \leq 0
\end{aligned}
$$

In order to complete the proof, the LaSalle Invariance Principle is invoked. All the trajectories converge to the largest invariant set $\bar{\Omega}$ in $\Omega=\{(\boldsymbol{q}, \boldsymbol{\omega}): \dot{V}=0\}=\{(\boldsymbol{q}, \boldsymbol{\omega}): \boldsymbol{\omega}=0\}$ In the invariant set, $J \dot{\boldsymbol{\omega}}=-\left[\begin{array}{lll}\alpha_{1} \lambda_{1} \rho_{1} q_{1} & \alpha_{2} \lambda_{2} \rho_{2} q_{2} & \alpha_{3} \lambda_{3} \rho_{3} q_{3}\end{array}\right]^{T}=0$ that is, $\bar{\Omega}$ is reduced to the origin. This ends the proof of the asymptotic stability of the closed loop system.

Remark 1. Since a quaternion and its negative represent the same physical angular position, there exist two equilibrium points: $\left(q_{0}= \pm 1, \boldsymbol{q}=0, \boldsymbol{\omega}=0\right)$. The equilibrium point $\left(q_{0}=-1, \boldsymbol{q}=0, \boldsymbol{\omega}=0\right)$ can be considered a repeller point (see Joshi et al. (1995)). However, it can be reached using the control law $\Gamma_{i}=-\alpha_{i} \sigma_{M_{i}}\left(\lambda_{i}\left[\omega_{i}-\rho_{i} q_{i}\right]\right)$ instead of (8). Therefore, applying

$$
\Gamma_{i}=-\alpha_{i} \sigma_{M_{i}}\left(\lambda_{i}\left[\omega_{i}+\operatorname{sign}\left(q_{0}\right) \rho_{i} q_{j}\right]\right)
$$

ensures that, of the two rotations of angle $\beta$ and $2 \pi-\beta$, the one of smaller angle is chosen. This can be demonstrated by adapting the previous proof.

Remark 2. Note that the stability analysis has been carried out considering the asymptotic condition (7). In the case that the asymptotic condition (6) is considered, the control law to be applied becomes

$$
\Gamma_{i}=-\alpha_{i} \sigma_{M_{i}}\left(\lambda_{i}\left[\omega_{i}+\operatorname{sign}\left(q_{e_{0}}\right) \rho_{i} q_{e_{j}}\right]\right)
$$

where $q_{e}$ represents the attitude error between the current orientation and the desired one.

\subsection{Some properties of the control law}

In practice, the measure of the angular velocity is generally obtained by rate gyros. These sensors can measure the angular velocity in an a priori specified range depending on the sensors and its technology. Therefore, the ability to maintain rotation about a rotational axis without exceeding a certain limit in the angular velocity arises essential. The control (8) allows to take into account the angular velocity constraints, i.e. slew rate limits. 
Let $\omega_{i_{\max }}$ denote the maximum magnitude angular velocity allowed for the rigid body manoeuvre about the $i$ axis. Let us consider the following state constraint

$$
\left|\omega_{i}\right| \leq \omega_{i_{\max }}
$$

then, one has the following result:

Property 1. (Slew rate constraints). The control law (8) globally asymptotically stabilizes the rigid body described by (3) and (5) to the origin. Moreover, the state constraint $\left|\omega_{i}\right| \leq \omega_{i_{\max }}$ is guaranteed to remain satisfied, for any $t_{1}>t_{0}$ if $\left|\omega_{i}\left(t_{0}\right)\right|>\omega_{i_{\max }}$ and for all $t$ if $\left|\omega_{i}\left(t_{0}\right)\right|<\omega_{i_{\max }}$, setting $\rho_{i}=\frac{\omega_{i_{\max }}}{2}$.

Proof. Assume that the system's initial condition lies anywhere in the state space where $\omega_{i}$ belongs to the ensemble $\Phi_{i}$ with $\Phi_{i}=\left\{\omega_{i}:\left|\omega_{i}\right| \leq 2 \rho_{i}\right\}$. From the proof of Theorem 1, one observes that if $\omega_{i}$ belongs to $\Phi_{i}, \omega_{i}$ remains in $\Phi_{i}$ for all time $t>0$. Therefore, if $\rho_{i}=\frac{\omega_{i_{\max }}}{2}$, one concludes that $\left|\omega_{i}\right| \leq 2 \rho_{i}=\omega_{i_{\max }}$, for all time $t>0$.

In several situations, the body angular velocities exceed the capability of the rate gyros (for instance, in the case that an external disturbance is present). The following propriety establish the robustness of the proposed control law towards bounded angular velocities measurements.

Property 2. (Robustness ). Let $\hat{\omega}_{i_{\max }}$ represent the maximum magnitude angular velocity that can be measured by the rate gyro along the $i$ axis. Let $\rho_{i}$ be chosen accordingly to property 1 , that is, $\rho_{i}=\frac{\hat{\omega}_{i_{\max }}}{2}$. Assume that the angular velocity exceeds the rate gyro limits, such as $\omega_{i}>\hat{\omega}_{i_{\max }}$. The control inputs (8) globally asymptotically stabilize the rigid body to the origin, in spite of measure saturation.

Proof. Assume that $\left|\omega_{i}\right|>\left|\hat{\omega}_{i_{\max }}\right|$ and $\operatorname{sign}\left(\omega_{i}\right)=$ $\operatorname{sign}\left(\hat{\omega}_{i_{\max }}\right)$ because to gyro rate saturation.

Consider the candidate Lyapunov function (9). The derivative of (9) after using (3) and (5) and the control law (8) is given by (10). Analyzing for $\dot{V}_{1}$ one gets,

$$
\dot{V}_{1}=-\alpha_{1} \omega_{1} \sigma_{M_{1}}\left(\lambda_{1}\left[\hat{\omega}_{1_{\max }}+\rho_{1} q_{1}\right]\right)+\kappa q_{1} \omega_{1}
$$

Since $\rho_{1}=\frac{\hat{\omega}_{1_{\max }}}{2}$, it follows that $\left|\hat{\omega}_{1_{\max }}+\rho_{1} q_{1}\right| \geq \frac{1}{2} \hat{\omega}_{1_{\max }}$. Because $\operatorname{sign}\left(\hat{\omega}_{1}\right)=\operatorname{sign}\left(\hat{\omega}_{1_{\max }}\right)$, from equation (24) and the norm condition on the quaternion, $\dot{V}_{1}$ takes the following form

$$
\begin{aligned}
\dot{V}_{1} & =-\alpha_{1} \omega_{1} \sigma_{M_{1}}\left(\lambda_{1}\left[\hat{\omega}_{1_{\max }}+\rho_{1} q_{1}\right]\right)+\kappa \omega_{1} q_{1} \\
& \leq-\alpha_{1}\left|\omega_{1}\right| \sigma_{M_{1}}\left(\lambda_{1}\left(\frac{1}{2} \hat{\omega}_{1_{\max }}\right)\right)+\kappa\left|\omega_{1}\right|
\end{aligned}
$$

Taking

$$
\kappa<\alpha_{1} \min \left(M_{1}, \lambda_{1} \frac{1}{2} \hat{\omega}_{1_{\max }}\right)
$$

one can assure the decrease of $V_{1}$. Consequently, $\omega_{1}$ enters $\Phi_{1}=\left\{\omega_{1}:\left|\omega_{1}\right| \leq 2 \rho_{1}\right\}$, that means $\left|\omega_{1}\right|<\left|\hat{\omega}_{1_{\max }}\right|$. Hence, the rate gyro is not saturated and the system evolves in the same way that the nominal system (no rate gyro saturation). The same argument is applied to $\dot{V}_{2}$ and $\dot{V}_{3}$.

Note that in addition to control torque constraints, the slew rate constraints and the robustness towards bounded angular velocities measurements are established.

\section{APPLICATION TO FOUR-ROTOR HELICOPTER}

In this section one shows that the control attitude strategy proposed previously can be stabilize in attitude a fourrotor helicopter. This mini helicopter (see Fig.1) has four fixed-pitch rotors mounted at the four ends of a simple cross frame. Given that the front and rear motors rotate counter-clockwise while the other two rotate clockwise, gyroscopic effects and aerodynamic torques tend to cancel in trimmed flight. The collective input (or throttle input) is the sum of the thrusts of each rotor $\left(f_{1}+f_{2}+f_{3}+f_{4}\right)$. Pitch movement $(\theta)$ is obtained by increasing (reducing) the speed of the rear motor while reducing (increasing) the speed of the front motor. The roll movement $(\phi)$ is obtained similarly using the lateral motors. The yaw movement $(\psi)$ is obtained by increasing (decreasing) the speed of the front and rear motors while decreasing (increasing) the speed of the lateral motors. This should be done while keeping the total thrust constant. In order to model the system dynamics, two frames are defined: the inertial frame $N\left(x_{n}, y_{n}, z_{n}\right)$ and the body-fixed frame $B\left(x_{b}, y_{b}, z_{b}\right)$ as shown in Fig.1. For more details the reader can refer to Pounds et al. (2002).

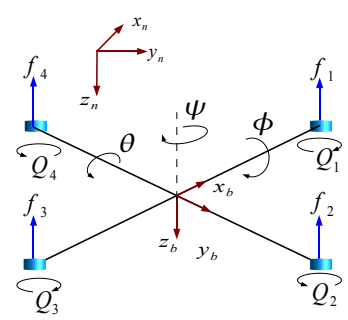

Fig. 1. Four-Rotor helicopter configuration: the inertial frame $N\left(x_{n}, y_{n}, z_{n}\right)$ and the body-fixed frame $B\left(x_{b}, y_{b}, z_{b}\right)$

According to Pounds et al. (2002) and section 2, the four-rotor helicopter model may be expressed in terms of quaternions as

$$
\begin{aligned}
\dot{p} & =v \\
\dot{v} & =\boldsymbol{g}^{N}-\frac{1}{m} C^{T}(q) \boldsymbol{T} \\
\dot{q} & =\frac{1}{2} \Xi(q) \boldsymbol{\omega} \\
J_{h} \dot{\boldsymbol{\omega}} & =-\boldsymbol{\omega} \times J_{h} \boldsymbol{\omega}-\Gamma_{G}+\Gamma
\end{aligned}
$$

$m$ denotes the mass of the helicopter, $\boldsymbol{g}^{N}$ is the vector of the gravity acceleration expressed in the $N$-frame. $p=(x, y, z)^{T}$ and $v=\left(v_{x}, v_{y}, v_{z}\right)^{T}$ represent the position and the linear velocity, respectively, of the origin of the $B$ frame expressed in the $N$-frame and $\boldsymbol{\omega}$ denotes the angular velocity of the helicopter expressed in the $B$-frame. $\Gamma \in \mathbb{R}^{3}$ is the vector of the control torques and $\boldsymbol{T}=\left[\begin{array}{lll}0 & 0 & T\end{array}\right]^{T}$ is the total thrust expressed in the $B$-frame. The attitude model of the four rotor aircraft differs from the general model (3)-(5) in the gyroscopic torques $\Gamma_{G} . \Gamma_{G} \in \mathbb{R}^{3}$ is the result of the rotational motion of the mini helicopter and the speed of the four rotors.

Equations (27)-(30) describe the 6 degrees of freedom of the system and can be separated into translational (27)(28) and rotational (29)-(30) motions. 


\subsection{Four-rotor Torque Control Design}

In order to stabilize the attitude of the four-rotor helicopter, equations (29)-(30) are used. The angular motion of the helicopter responds to control torques arising from the linear mapping of the rotation speed of the rotors. Hence, the maximum airframe control torque depends of the much higher rotation speed capability of the motors that are used.

In this application, the rotors are driven by DC permanent magnet motors which support a maximum voltage of $9 \mathrm{~V}$. When this voltage is applied to the motor the rotation speed reaches $s_{i, \max }=260 \mathrm{rad} / \mathrm{sec}$. Consequently, from the motor characteristics mentioned above and the from aerodynamical characteristics of the propeller, the maximum torque that is applied to influence the helicopter rotational motion is given by

$$
\bar{\Gamma}_{1}=0.40 \mathrm{Nm} \bar{\Gamma}_{2}=0.40 \mathrm{Nm} \bar{\Gamma}_{3}=0.15 \mathrm{Nm}
$$

In order to avoid unwanted damages in the actuators and to maximize its effectiveness, the bounded attitude control presented in the previous section is applied to the subsystem (29)-(30).

Lemma 1. Consider the four-rotor helicopter rotational dynamics described by (29) and (30) with the following bounded control inputs

$$
\begin{aligned}
& \Gamma_{1}=-\alpha_{1} \sigma_{M_{\phi}}\left(\lambda_{1}\left[\omega_{1}+\rho_{1} q_{1}\right]\right) \\
& \Gamma_{2}=-\alpha_{2} \sigma_{M_{\theta}}\left(\lambda_{2}\left[\omega_{2}+\rho_{2} q_{2}\right]\right) \\
& \Gamma_{3}=-\alpha_{3} \sigma_{M_{\psi}}\left(\lambda_{3}\left[\omega_{3}+\rho_{3} q_{3}\right]\right)
\end{aligned}
$$

where $\sigma_{M_{\phi, \theta, \psi}}$ are saturation functions such as

$$
M_{\phi} \geq 3 \lambda_{1} \rho_{1}, M_{\theta} \geq 3 \lambda_{2} \rho_{2}, M_{\psi} \geq 3 \lambda_{3} \rho_{3}
$$

with $\alpha_{i}, \lambda_{i}$ and $\rho_{i}$ positive parameters. Then the inputs (31) globally stabilize the four-rotor helicopter to the origin $\left(q_{0}=1, \boldsymbol{q}=0\right.$ and $\left.\boldsymbol{\omega}=0\right)$.

$M_{\phi, \theta, \psi}$ and $\alpha_{i}$ are chosen to satisfy the following equations

$$
\bar{\Gamma}_{1}=\alpha_{1} M_{\phi} \bar{\Gamma}_{2}=\alpha_{2} M_{\theta} \bar{\Gamma}_{3}=\alpha_{3} M_{\psi}
$$

Proof. The steps of the proof are identical to the ones of Theorem 1. Indeed, the only difference lies in the vector $\Gamma_{G}$ that adds a term canceled because of the relation:

$$
\boldsymbol{\omega}^{T} \Gamma_{G}=\boldsymbol{\omega}^{T}\left(\boldsymbol{\omega} \times z_{b}\right) \sum_{i=1}^{4} J_{r}(-1)^{i+1} s_{i}=0
$$

where $J_{r}$ represents the inertia of the rotor.

\section{EXPERIMENTAL RESULTS}

The aim of this section is to show the effectiveness of the proposed control approach. For this purpose, an attitude stabilization in real-time was performed on the four-rotor mini helicopter prototype of "gipsa-lab". This prototype is based in the mechanical structure (airframe, motors and blades) of the Draganflyer III developed by RC Toys. For the elaboration of real-time processing board, it was used an embedded microcontroller $(\mu C)$ C8051F022 manufactured by Silicon Laboratories, an inertial measurement unit (3DM-G from Microstrain), a bi-directional communication unit (SPM2-433-28 from Radiometrix), a power module to drive the motors (MOSFET transistors) and four optical sensors attached to the ends of the mechanical frame to measure the rotor speed. The power is supplied by a 9 Volts Lithium Polymer battery. On the other hand, a communication unit (SPM2-433-28 from Radiometrix) is linked to a $\mathrm{PC}$, in order to provide and to obtain the process data. The $\mu C$ acquires the angular velocity and attitude (quaternion) provided by the IMU and it obtains the desired attitude incoming from the ground station. Thus, the $\mu C$ executes the attitude control law and computes the PWM level to control the four motors. Optionally, the embedded system provides the process data to the ground station, in order to monitor the experiment. The attitude control loop runs at $f_{s}=76 \mathrm{~Hz}$, this is due to IMU constraints.

Three experiments have been accomplished. At the first and second case the goal was the attitude stabilization at the origin $\left(q_{d}=\left[\begin{array}{llll}1 & 0 & 0 & 0\end{array}\right]\right.$. i.e. $\left.\phi_{d}=\theta_{d}=\psi_{d}=0\right)$. In the third case a command following was performed. The desired thrust is fixed to $T=m g=4.59 \mathrm{~N}$ and the maximum torque frame that can be applied is $\bar{\Gamma}=$ $\left[\begin{array}{lll}0.40 & 0.40 & 0.15\end{array}\right]^{T} \mathrm{Nm}$. The tuning parameters of the control input are as follows: $\alpha_{i}=1, \rho_{1}=\rho_{2}=2.1$, $\rho_{3}=0.87$. The Euler angles are shown, nevertheless, the algorithm is carried out in the quaternion formalism.

\section{- Stabilization}

The first experiment tests the control capabilities to stabilize the system at the origin. The obtained results are depicted in Fig.2 - Fig.4. According to the angles trajectories, the stabilization is achieved in $1.5 \mathrm{sec}$, which is a very suitable time. Moreover, the constraints in the control signal are largely satisfied.

\section{- Disturbance rejection}

For the second experiment, the robustness of the proposed controller towards disturbance rejection has been tested. The disturbances along each axis (in both directions) are introduced into the system after to achieve the attitude stabilization. As can be seen in the Fig. 5 and Fig. 6, the disturbance produces a large error in the yaw angle as well as in angular velocity $\omega_{z}$. Consequently, the control signal $\Gamma_{3}$ reaches its limit $( \pm 0.15 N \cdot m)$ (see Fig. 7) and takes action on the system to overcome the disturbances. A similar rejection disturbance is observed for the pitch and the roll angle, while only feasible control signals are applied to the system. This study case shows that the controller proposed in this paper is robust with respect to external disturbances, and the more important, the control law maximize the effectiveness of the actuators without endanger the system stability. The robustness property is essential in real missions where aerodynamic forces and others factors are presents.

\section{- Command following}

The third experiment deals with the attitude tracking. It is observed (see Fig. 8) that the controller is able to following the reference signal $r_{t}=9^{\circ}+9^{\circ} \sin \left(\frac{15}{\pi} t\right)$ along the axis $z_{b}$. In the case that step signals are considered to be tracked, the problem results in an stabilization problem (see remark 2 ). This experiment enables to show the performance of the proposed control scheme to tracking a reference attitude, even thought the controller was designed for stabilization. 


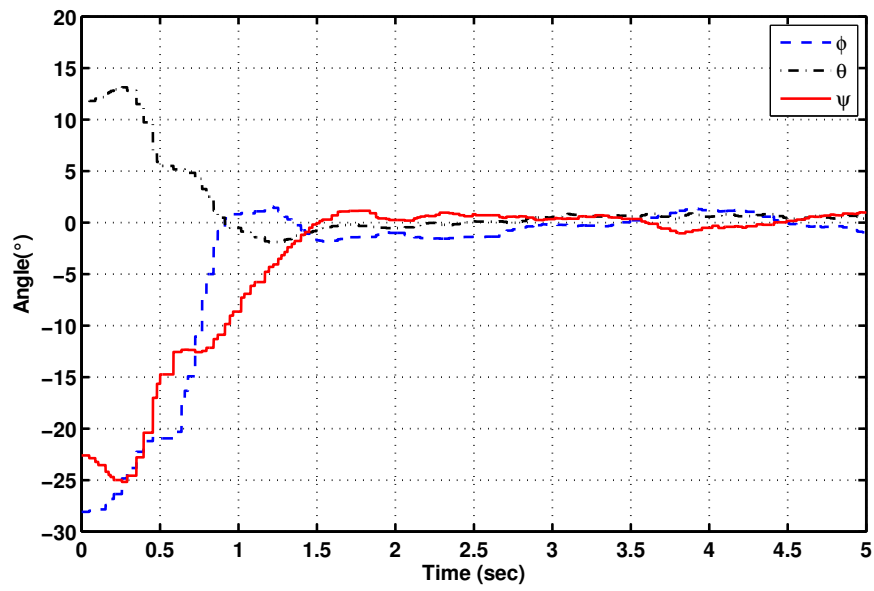

Fig. 2. Stabilization: The convergence of the roll, pitch and yaw angles with initial conditions $\phi=-28^{\circ}, \quad \theta=$ $13^{\circ}, \psi=-23^{\circ}$.

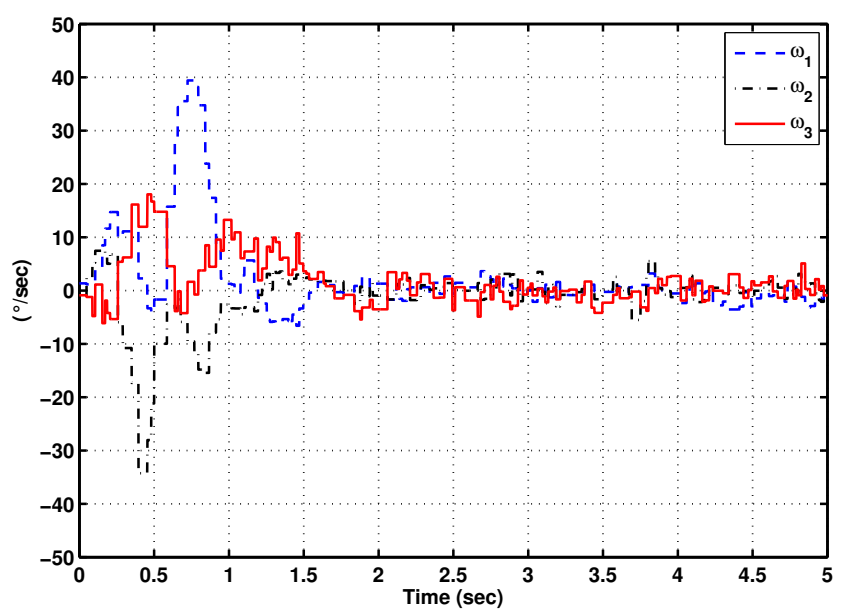

Fig. 3. Stabilization: The evolution of the angular velocity.

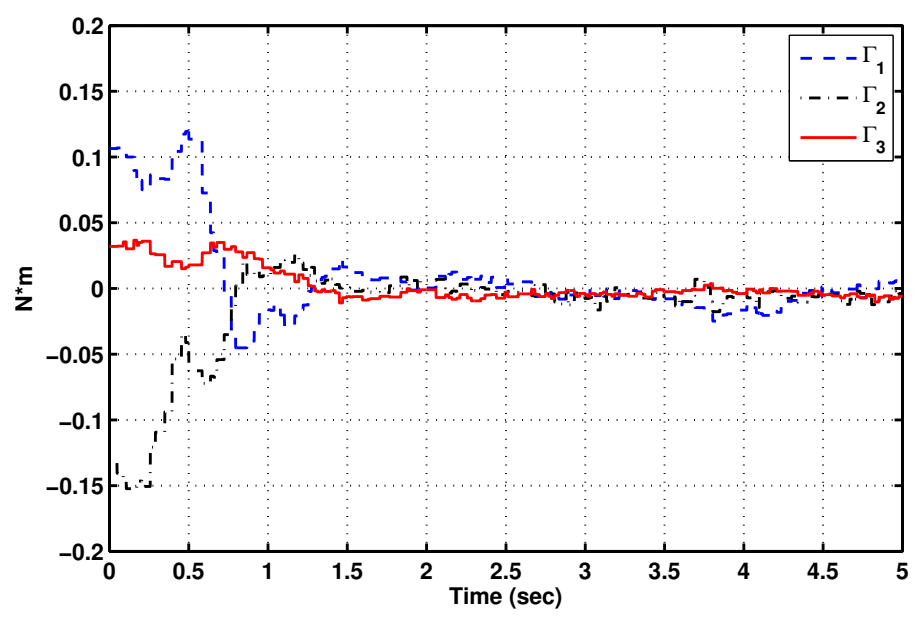

Fig. 4. Stabilization: The bounded control torque.

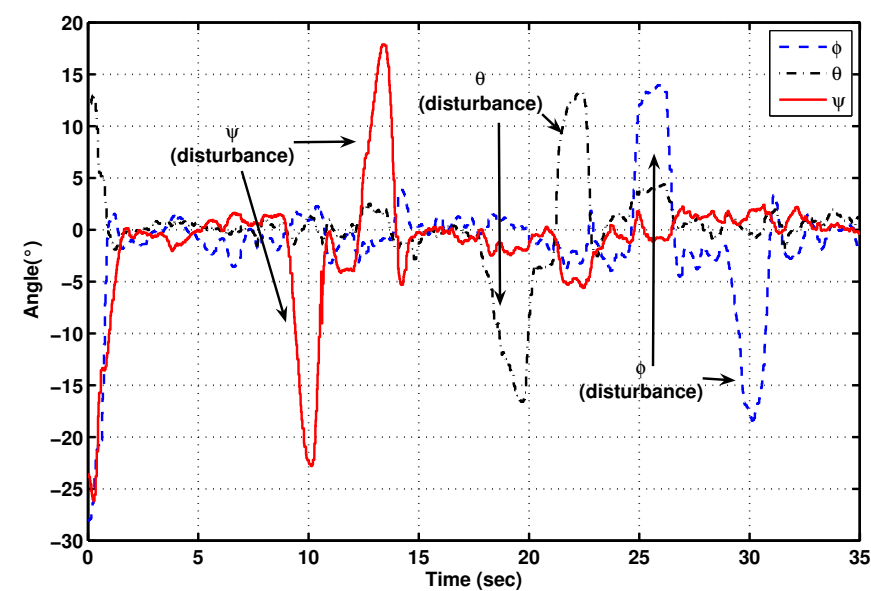

Fig. 5. Disturbance rejection: The convergence of the roll, pitch and yaw angles, while the system is subject to external disturbances.

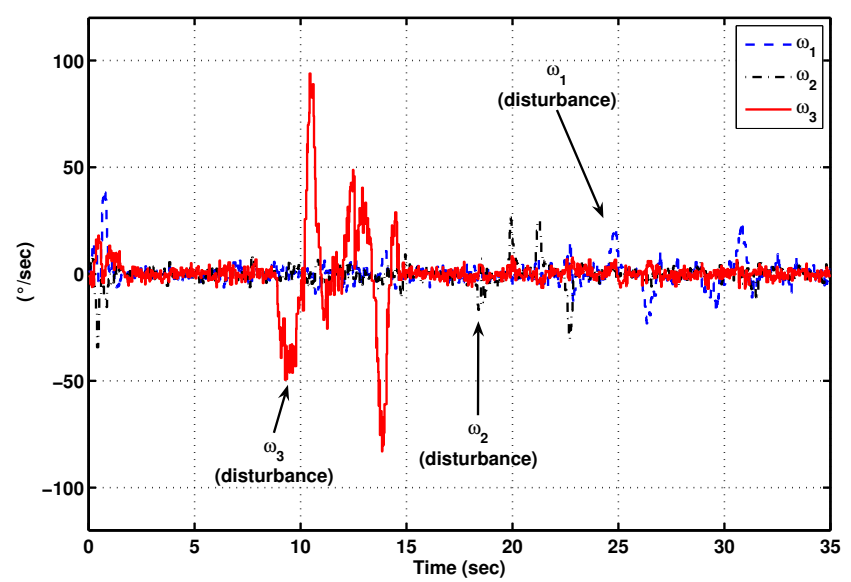

Fig. 6. Disturbance rejection: The evolution of the angular velocity, while the system is subject to external disturbances.

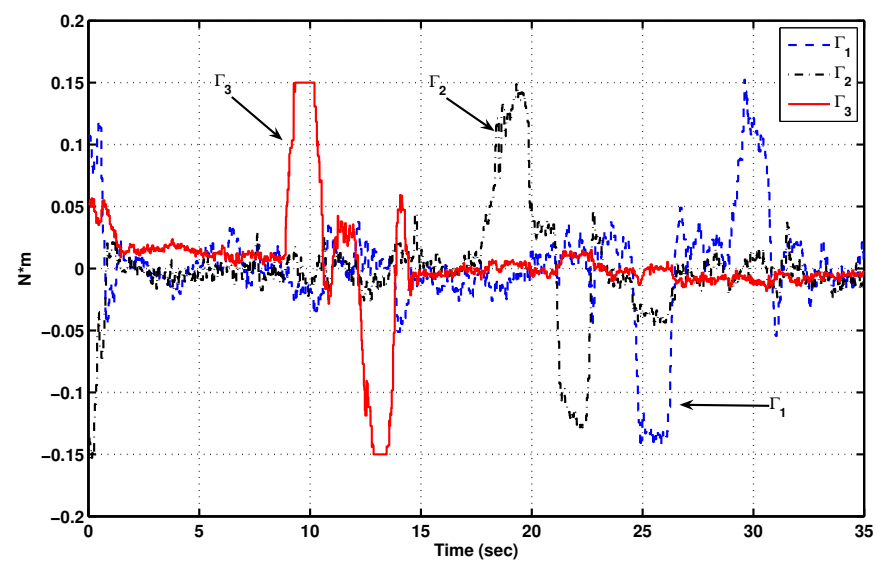

Fig. 7. Disturbance rejection: The bounded control torque signal, while the system is subject to external disturbances. The control constraints are satisfied. 


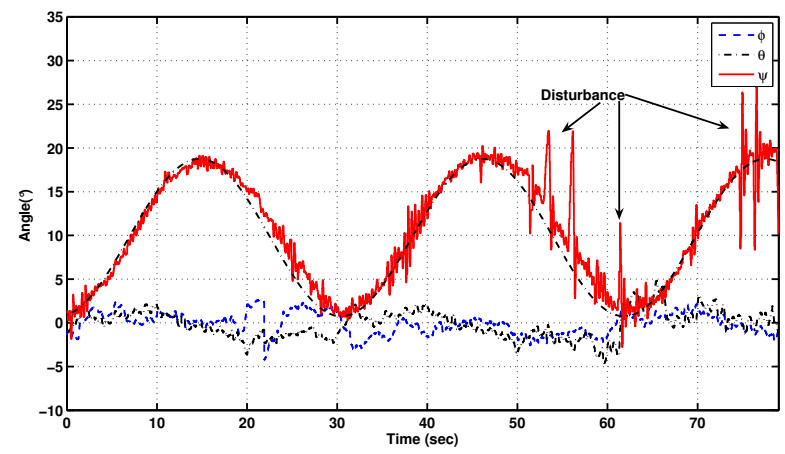

Fig. 8. Tracking: The evolution of the roll, pitch and yaw angles, while the system tracks a sinus signal along the axis $z_{b}$.

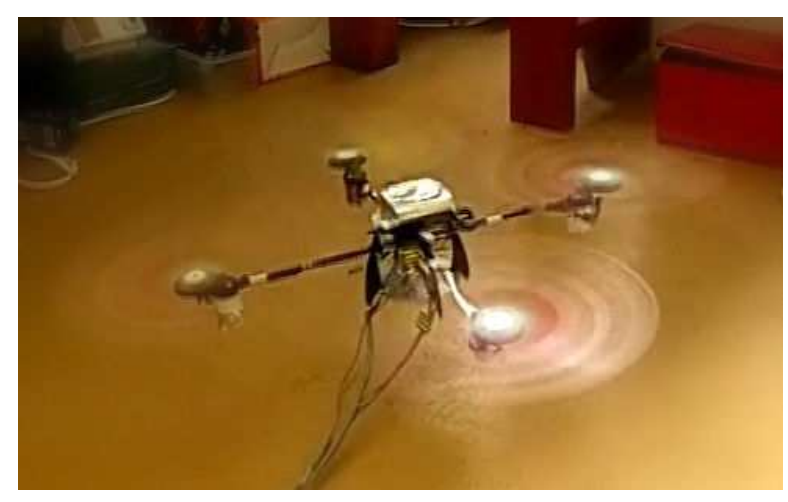

Fig. 9. The four-rotor min-helicopter in flight.

\section{CONCLUSIONS}

In this paper, a new bounded control law for the global stabilization of a rigid body is proposed. The objective of this control approach is to maximize the effectiveness of the actuators without taking a risk in the system stability. The control design takes into account the slew rate limits, avoiding possible rate gyro saturation. The presented scheme control is especially simple. It is based on nested saturation approach and the attitude is parameterized by the unit quaternion. The proposed approach is extended to the stabilization of a four-rotor helicopter. Thus, the implementation in real-time is achieved. Several tests are performed showing the performance in term of settling time, disturbance rejection and trajectory tracking. Remain to compare the proposed approach with other control schemes. However, owing to simplicity, the proposed control law is suitable for application where on-board computational resources are challenging.

\section{REFERENCES}

C. Belta. On controlling aircraft and underwater vehicles. In IEEE International Conference on Robotics and Automation, 2004.

J.D. Boskovic, S.-M. Li, and R.K. Mehra. Robust stabilization of spacecraft in the presence of control input saturation using sliding mode control. In AIAA Guidance, Navigation, and Control Conference and Exhibit, 1999.
S. Bouabdallah and R. Siegwart. Backstepping and sliding-mode techniques applied to an indoor micro quadrotor. In IEEE International Conference on Robotics and Automation, 2005.

S. Bouabdallah, A. Noth, and R. Siegwart. PID vs LQ control techniques applied to an indoor micro quadrotor. In International Conference on Intelligent Robots and Systems, 2004.

O. Fjellstad and T. Fossen. Quaternion feedback regulation of underwater vehicles. In 3rd IEEE Conference on Control Application, 1994.

J.F. Guerrero-Castellanos, A. Hably, N. Marchand, and S. Lesecq. Bounded attitude stabilization: Application on four rotor helicopter. In IEEE International Conference on Robotics and Automation, 2007.

O. Hegrenas, J.T. Gravdahl, and P. Tondel. Attitude control by means of explicit model predictive control, via multi-parametric quadratic programming. In American Control Conference, 2005.

S.M. Joshi, A.G. Kelkar, and J.T. Wen. Robust attitude stabilization of spacecraft using nonlinear quaternion feedback. IEEE Transactions on Automatic Control, 40 (10):1800-1803, 1995.

R. Kristiansen and P. J. Nicklasson. Satellite attitude control by quaternion-based backstepping. In American Control Conference, 2005.

M. Krstić and P. Tsiotras. Inverse optimal stabilization of a rigid spaceraft. IEEE Transactions on Automatic Control, 44(5):1042-1049, 1999.

M. Osipchuck, K.D. Bharadwaj, and K.D. Mease. Achieving good performance in global attitude stabilization. In American Control Conference, 1997.

P. Pounds, R. Mahony, P. Hynes, and J. Roberts. Design of a four-rotor aerial robot. In Australian Conference on Robotics and Automation, 2002.

S.L. Scrivener and R.C. Thompson. Survey of timeoptimal attitude maneuvers. Journal of Guidance, Control and Dynamics, 17(2):225-233, 1994.

M.D. Shuster. A survey of attitude representations. Journal of the astronautical sciences, 41(4):439-517, 1993.

C. Song, S.-J. Kim, S.-H. Kim, and H.S. Nam. Robust control of the missile attitude based on quaternion feedback. Control Engineering Practice, 14:811-818, 2005 .

A. Tayebi and S. McGlivary. Attitude stabilization of a vtol quadrotor aircraft. IEEE Transactions on Control Systems Technology, 14(3):562-571, 2006.

A.R. Teel. Global stabilization and restricted tracking for multiple integrators with bounded controls. Systems $\&$ Control Letters, 18:165-171, 1992.

P. Tsiotras and J. Luo. Control of underactuated spacecraft with bounded inputs. Automatica, 36(8):11531169, 2000.

J.T. Wen and K. Kreutz-Delgado. The attitude control problem. IEEE Transactions on Automatic Control, 36 (11):1148-1162, 1991.

J.T. Wen, S. Seereeram, and D.S. Bayard. Nonlinear predictive control applied to spacecraft attitude control. In American Control Conference, 1997.

B. Wie, H. Weiss, and A. Arapostathis. Quaternion feedback regulator for spacecraft eigenaxis rotations. Journal of Guidance, Control and Dynamics, 12(3):375$380,1989$. 